\title{
THE INFLUENCE OF THE GRAIN STRUCTURE SIZE ON MICROSTRUCTURALLY SHORT CRACKS
}

\author{
Revised and extended version of the paper \\ presented at the ICONE-16, May 11-15, 2008, Orlando, Fl., USA \\ Igor Simonovski, Leon Cizelj \\ »Jožef Stefan« Institute, \\ Reactor Engineering Division \\ Jamova cesta 39, \\ SI-1000 Ljubljana \\ Slovenia \\ Email: LeonCizelj@ijs.si
}

\section{KEYWORDS}

short cracks, crack tip opening displacement, polycrystalline material; crystal plasticity

\section{ABSTRACT}

The dominant processes in the initialization and propagation of microstructurally short cracks include microstructural features such as crystallographic orientations of grains, grain boundaries, inclusions, voids, material phases, etc. The influence of the microstructural features is expected to vanish with distance from the crack tip. Also, the influence of the nearby microstructural features is expected to be smaller for a long than for a small crack. Finally, a crack of sufficient length can be modeled using classical fracture mechanics methods. In this paper the approach to estimate the crack length with vanishing influence from the microstructural feature is proposed. To achieve this, a model containing a large number of randomly sized, shaped and oriented grains is employed. The random grain structure is modeled using a Voronoi tessellation. A series of cracks of lengths from about 1 to 7 grain lengths are inserted into the model, extending from a grain at the surface towards the interior of the model. The crack tip opening displacements (CTOD) are estimated and statistically analyzed for a series of random crystallographic orientation sets assigned to the grains adjacent to the crack. Anisotropic elasticity and crystal plasticity constitutive models are employed at the grain size scale.

It is shown that the standard deviation of the crack tip opening displacement decreases from about $20 \%$ for a short surface crack embedded within a single grain to about $7 \%$ for a surface crack extending through 7 grains. From the engineering point of view, a crack extending through less than about 10 grains sizes is therefore considered to strongly depend on the neighboring microstructural features. 


\section{NOMENCLATURE}

$C_{i j k l}$

$D_{i j k l}$

$\dot{a}^{(\alpha)}$

$\dot{g}^{(\alpha)}$

$g^{(\alpha)}$

$h_{0}$

$h_{\alpha \alpha}$

$h_{\alpha \beta}$

$m_{i}^{(\alpha)}$

$n$

$q$

$s_{j}^{(\alpha)}$

$\alpha$

$\dot{\gamma}^{(\alpha)}$

$\varepsilon_{i j}$

$\dot{\varepsilon}_{k l}$

$\dot{\varepsilon}_{k l}^{p}$

$\theta$

$\sigma_{i j}$

$\dot{\sigma}_{i j}$

$\tau_{0}$

$\tau^{(\alpha)}$

AISI

CTOD

ITER

LEFM stiffness tensor

compliance tensor

strain rate in slip system $\alpha$

current strain hardened state in slip system $\alpha$

current strength in slip system $\alpha$

initial hardening modulus

self-hardening moduli

slip-hardening moduli

slip plane normal

strain rate sensitivity parameter

hardening factor

slip direction

grain's crystallographic orientation

slipping rate in slip system $\alpha$

strain tensor

strain rate tensor

plastic strain rate tensoR

crack direction

stress tensor

stress rate tensor

yield stress

Taylor's resolved shear stress

American Iron and Steel Institute

Crack tip opening displacement

International Thermonuclear Experimental Reactor

linear elastic fracture mechanics 


\section{INTRODUCTION}

Ageing and damage in materials could play a significant role in the long term safe operation of nuclear power plants. Vast knowledge about ageing and damage has accumulated over the years. There are however some remaining issues calling for better explanation. These include initialization and propagation of microstructurally short cracks, which account for a rather significant proportion of a component's life time.

The dominant processes in the initialization and propagation of microstructurally short cracks include microstructural features such as crystallographic orientations of grains, grain boundaries, inclusions, voids, material phases, etc. [1][2][3]. It has been noted for example that the crack growth rate can be decreased when the crack approaches the grain boundary [3][4] and [5]. Also, short cracks tend to closely follow the slip plane. The crack therefore changes the direction when crossing the grain boundary [6]. This often results in zigzag patterns [7]. Different crystallographic orientations of the grains may also accelerate, decelerate or even arrest the crack growth [8][9]. The crack tip loading in the highly variable deformation field caused by the inhomogeneous grain structure is therefore generally mixedmode with strong shear component. The size of the plastic zone around the crack tip grows comparable to the crack length already at relatively small loads, therefore severely thwarting the applicability of linear elastic fracture mechanics.

In this paper the approach to estimate the crack length with vanishing influence from the microstructural feature is proposed. To achieve this, a model containing a large number of randomly sized, shaped and oriented grains is employed. The random grain structure is modeled using a Voronoi tessellation. A series of transgranular cracks of lengths from about 1 to 7 grain lengths are inserted into the model, extending from a grain at the surface and kinking across grain boundaries towards the interior of the model. Anisotropic elasticity and crystal plasticity constitutive models are employed at the grain size scale with grain boundaries modeled simply as a discontinuity in the lattice orientation between neighboring grains. Dislocations are not modeled, although it is acknowledged that they are important, especially near the grain boundaries [10][11]. The proposed approach builds on the previous work on the modeling of short cracks by the authors [12] [13].

Crack tip opening displacements (CTOD) values are calculated for a series of stationary cracks under monotonic uniaxial tensile load. Different sets of random crystallographic orientations are applied to enable statistical analysis of the variability of CTOD values due to the surrounding crystallographic orientations.

The material properties used in this paper are typical for the AISI 316L austenitic stainless steel, which is extensively used in nuclear industry (e.g. pipes) and has also been selected for a number of components of the International Thermonuclear Experimental Reactor ITER (vacuum vessel and ports, blanket shield modules, thin walled tubes for the first wall, cooling manifolds, diverter body etc.[14]. 


\section{MODEL}

The structural model includes a planar rectangular polycrystalline aggregate with 5027 randomly sized and shaped grains, outlined in Figure 1. The finite elements are omitted from Figure 1 to minimize the clutter. The planar grain structure is approximated using a planar Voronoi tessellation, generated using the VorTESS code [15]. In order to facilitate the finite element meshing, a Voronoi tessellation with reasonably small aspect ratios of cord lengths has been selected [16].

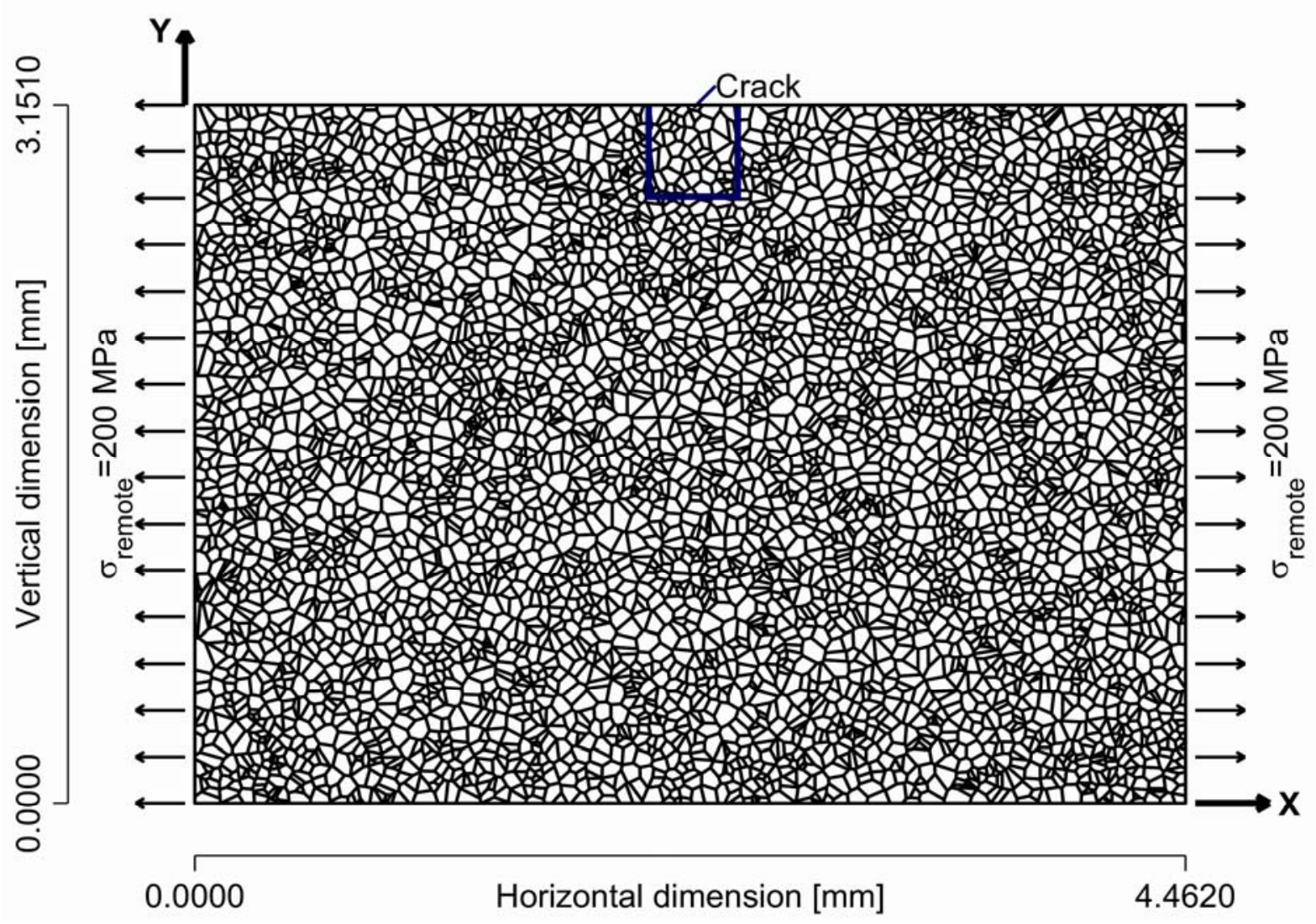

Figure 1 The outline of the finite element model.

The applied loading and boundary conditions are illustrated in Fig. 1. The left and right edges are monotonically loaded in macroscopic uniaxial tension (with zero shear traction) up to a maximum load of $200 \mathrm{MPa}$, which is equivalent to about $80 \%$ of the yield strength of the material $(240 \mathrm{MPa})$. This load is sufficient to trigger significant slip systems activity in all analyzed cases. The upper and lower edges are traction free. Prevention of rigid body movement is also imposed.

Each grain is assumed to behave as a randomly oriented monocrystal governed by the anisotropic elasticity and crystal plasticity models, described in section 2.1 Constitutive model below. Crystallographic orientations are defined by:

- Setting the angle between the crystallographic [100] direction and the macroscopic X direction of all crystals in the model to $135^{\circ}$, as shown in Figure 2 . This results in a planar slip system model compatible with the planar macroscopic model: the projections of the primary and conjugate slip planes are depicted in Figure 3. 
- Setting random rotation of the lattice in individual grains about the global Z-axis. The angle of this random rotation is hereafter referred to as crystallographic orientation $\alpha$. Fig. 3.
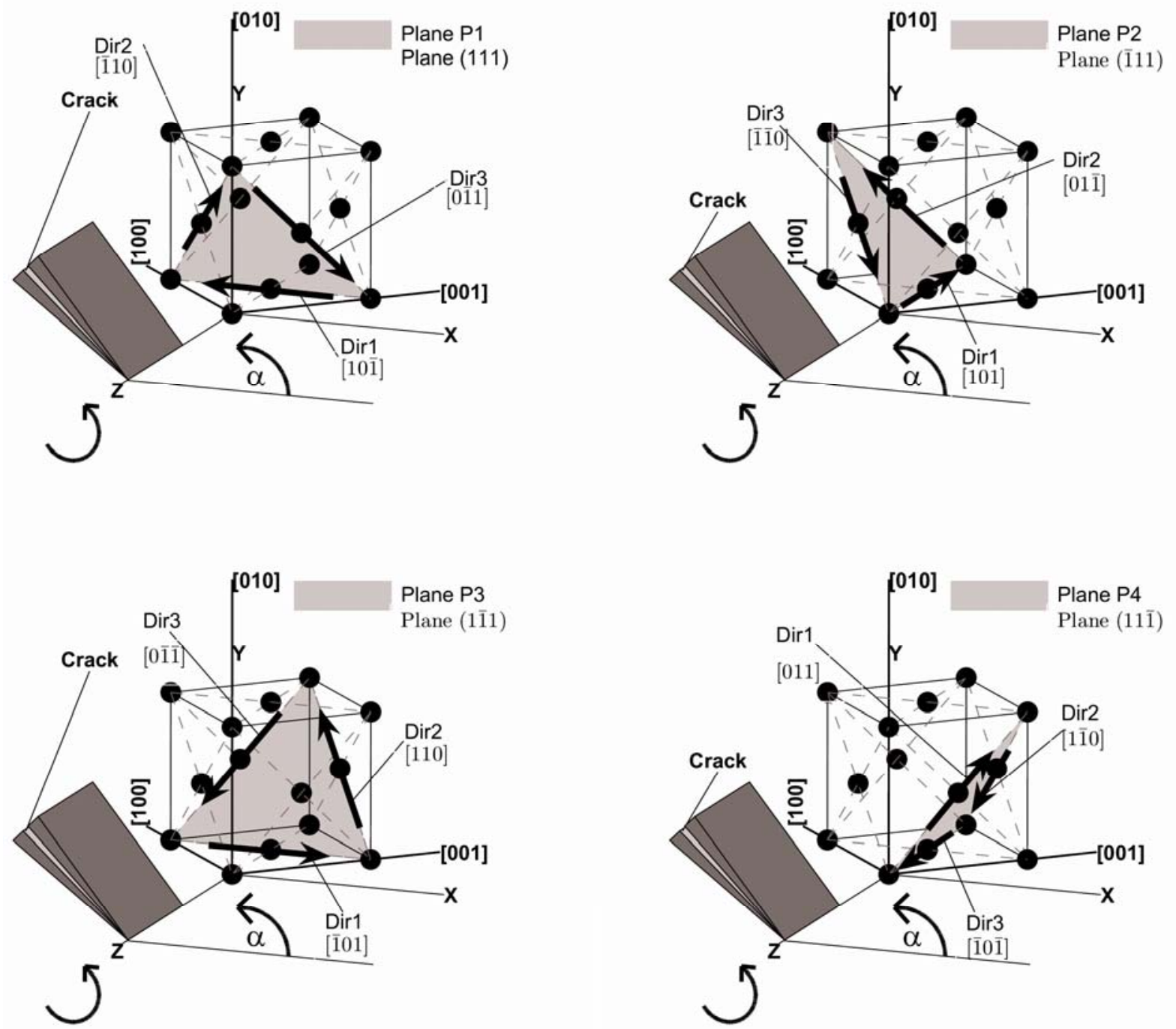

Figure 2 Relation between the slip systems of a face centered cubic crystal and the crack for crystallographic orientation $\alpha=0^{\circ}$ and crack direction $\theta=315^{\circ}$.

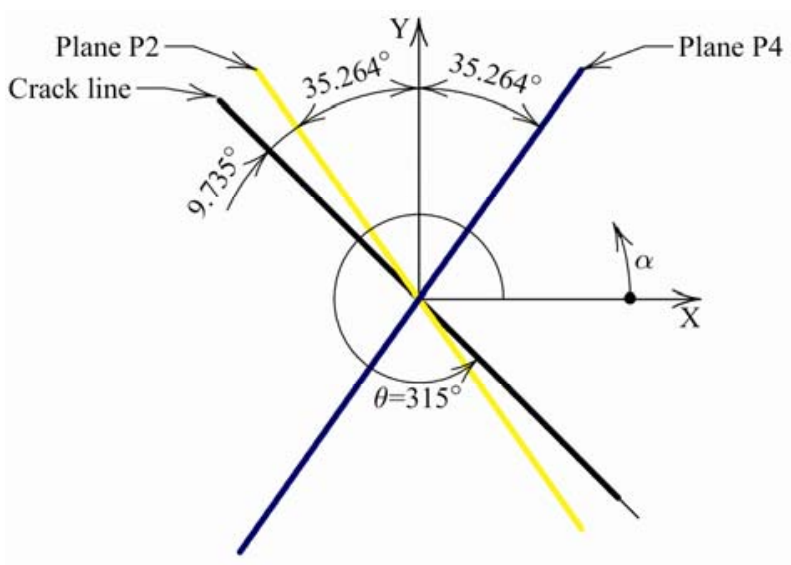

Figure 3 Angles between the slip planes and the crack plane for crystallographic orientation $\alpha=0^{\circ}$ and crack direction $\theta=315^{\circ}$. 
A set of short surface cracks of different lengths, all of them representative of a Stage I fatigue cracks, are introduced into the model at the free surface as denoted at the middle upper part of Figure 1. Detailed description follows in Section 2.3 Layout of cracks.

\subsection{Constitutive model}

The main features of the elasto-plastic constitutive model of monocrystal are briefly explained below. Each crystal grain in the polycrystalline aggregate is assumed to behave as an anisotropic continuum. Random orientations of crystal lattice differ from grain to grain. Constitutive relations in linear elasticity are given by the generalized Hooke's law:

$$
\sigma_{i j}=C_{i j k l} \cdot \varepsilon_{k l},
$$

where $\sigma_{i j}$ represents the second rank stress tensor, $C_{i j k l}$ represents the fourth rank stiffness tensor and $\varepsilon_{i j}$ represents the second rank strain tensor. Indices $i, j, k$ and $l$ are running from 1 to 3 . The inverse of the stiffness tensor, the compliance tensor $D_{i j k l}$ is defined as:

$$
\varepsilon_{i j}=D_{i j k l} \cdot \sigma_{k l} \text {. }
$$

Crystal plasticity used in the proposed model follows the pioneering work of Taylor [17], and Hill and Rice [18]. It is assumed that the plastic deformation is a result of crystalline slip only. The crystalline slip is driven by the resolved shear stress $\tau^{(\alpha)}$ ) [19]:

$$
\tau^{(\alpha)}=m_{i}^{(\alpha)} \cdot \sigma_{i j} \cdot s_{j}^{(\alpha)},
$$

where $\alpha$-th slip system is defined by a combination of slip plane (determined by normal $m_{i}^{(\alpha)}$ ) and slip direction $\left(s_{j}^{(\alpha)}\right)$. The number of slip systems and their orientations depend on the crystal lattice (e.g., 48 in the body and 12 in the face centered cubic lattice). The stress rate can be defined as:

$$
\dot{\sigma}_{i j}=C_{i j k l} \cdot\left(\dot{\varepsilon}_{k l}-\dot{\varepsilon}_{k l}^{p}\right)=C_{i j k l} \cdot\left(\dot{\varepsilon}_{k l}-\sum_{\alpha} \frac{1}{2} \dot{\gamma}^{(\alpha)}\left(s_{i}^{(\alpha)} m_{j}^{(\alpha)}+s_{j}^{(\alpha)} m_{i}^{(\alpha)}\right)\right) \text {, }
$$

where $\dot{\sigma}_{i j}$ is the stress rate tensor, $\dot{\varepsilon}_{k l}$ is the strain rate tensor, $\dot{\varepsilon}_{k l}^{p}$ is the plastic strain rate tensor and $\dot{\gamma}^{(\alpha)}$ is the slipping rate of the $\alpha$-th slip system. The slipping rate $\dot{\gamma}^{(\alpha)}$ is assumed to be governed by the resolved shear stress $\tau^{(\alpha)}$ in a visco-plastic framework [20]:

$$
\dot{\gamma}^{(\alpha)}=\dot{a}^{(\alpha)}\left(\frac{\tau^{(\alpha)}}{g^{(\alpha)}}\right)\left(\left|\frac{\tau^{(\alpha)}}{g^{(\alpha)}}\right|\right)^{n-1},
$$

where $\dot{a}^{(\alpha)}$ is reference strain rate, $n$ the strain rate sensitivity parameter and $g^{(\alpha)}$ the current strain hardened state of the crystal. In the limit as $n$ approaches infinity this power law approaches that of a rate-independent material. The current strain hardened state $g^{(\alpha)}$ is defined by:

$$
\dot{g}^{(\alpha)}=\sum_{\beta} h_{\alpha \beta} \dot{\gamma}^{(\beta)}
$$

where $h_{\alpha \beta}$ are the slip hardening moduli. Different proposals of hardening moduli could be found in literature, most of them relying on empirical models. Peirce et al. [21] hardening law is used in numerical example. Self- $\left(h_{\alpha \alpha}\right)$ and latent-hardening moduli $\left(h_{\alpha \beta}\right)$ are defined as:

$$
h_{\alpha \alpha}=h(\gamma)=h_{0} \operatorname{sech}^{2}\left|\frac{h_{0} \gamma}{\tau_{S}-\tau_{0}}\right|, h_{\alpha \beta}=q h(\gamma),(\alpha \neq \beta) \text {, }
$$


where $h_{0}$ is the initial hardening modulus, $\tau_{0}$ the yield stress, which equals the initial value of current strength $g^{(\alpha)}(0), \tau_{S}$ the break-through stress where large plastic flow initiates, $\gamma$ the cumulative slip and $q$ is hardening factor.

A user subroutine[20], which incorporates anisotropic elasticity and crystal plasticity with finite-strain and finite-rotation formulations, was used in the commercially available finite element code ABAQUS.

\subsection{Material parameters}

The following elastic constants for the AISI $316 \mathrm{~L}$ face centered cubic crystal are used: $C_{i i i i}=$ $163680 \mathrm{MPa}, C_{i i j j}=110160 \mathrm{MPa}, C_{i j i j}=100960 \mathrm{MPa}$ [22]. Crystal plasticity parameters have been optimized from the macroscopic plastic response of AISI 316L polycrystal [22]: $h_{0}=$ $330 \mathrm{MPa}, \tau_{s}=270 \mathrm{MPa}, \tau_{0}=90 \mathrm{MPa}, n=55, q=1.0$ and $a^{(\alpha)}=0.001$. With these parameters the proposed plain strain model is deemed sufficiently accurate to provide a correct qualitative representation of the macroscopic response[22]. The reference calculations with isotropically elastic and plastic material models have been carried out using Youngs modulus of $192 \mathrm{GPa}$, Poisson ratio of 0.3 , yield strength of $240 \mathrm{MPa}$ and hardening modulus of $1118 \mathrm{MPa}$.

\subsection{Layout of cracks}

A set of short surface cracks of different lengths, all of them representative of a Stage I fatigue crack, are introduced into the model (Figure 4). The shortest crack is completely embedded in the grain No. 4854. The crack is placed in the slip plane denoted as P2 in Figure 2. Increasing the crack length requires crossing the grain boundaries and kinking of the crack direction, which is assumed to follow the slip plane also in the newly damaged grain.

In the model, the crack path was assumed arbitrarily, keeping in mind that the growing crack would finally tend towards the pure mode I loading. The assumed path of the crack and the assumed coincidence of the crack with slip plane P2 (Figure 2) also required that the orientations of the lattice in grains along the crack path are fixed accordingly. It follows from Figure 2 that the crack with the direction $\theta$, measured in the counter clockwise direction, will coincide with the slip plane P2 in the lattice with orientation $\alpha$ when:

$$
\theta=90^{\circ}+25.264^{\circ}+\alpha+180^{\circ} \text {. }
$$

Table 1 lists the crystallographic orientations $\alpha$ and crack directions $\theta$ for all grains along the assumed crack path. The orientation of all remaining grains in the model is random with uniform distribution in the range between 0 and $2 \pi$. 

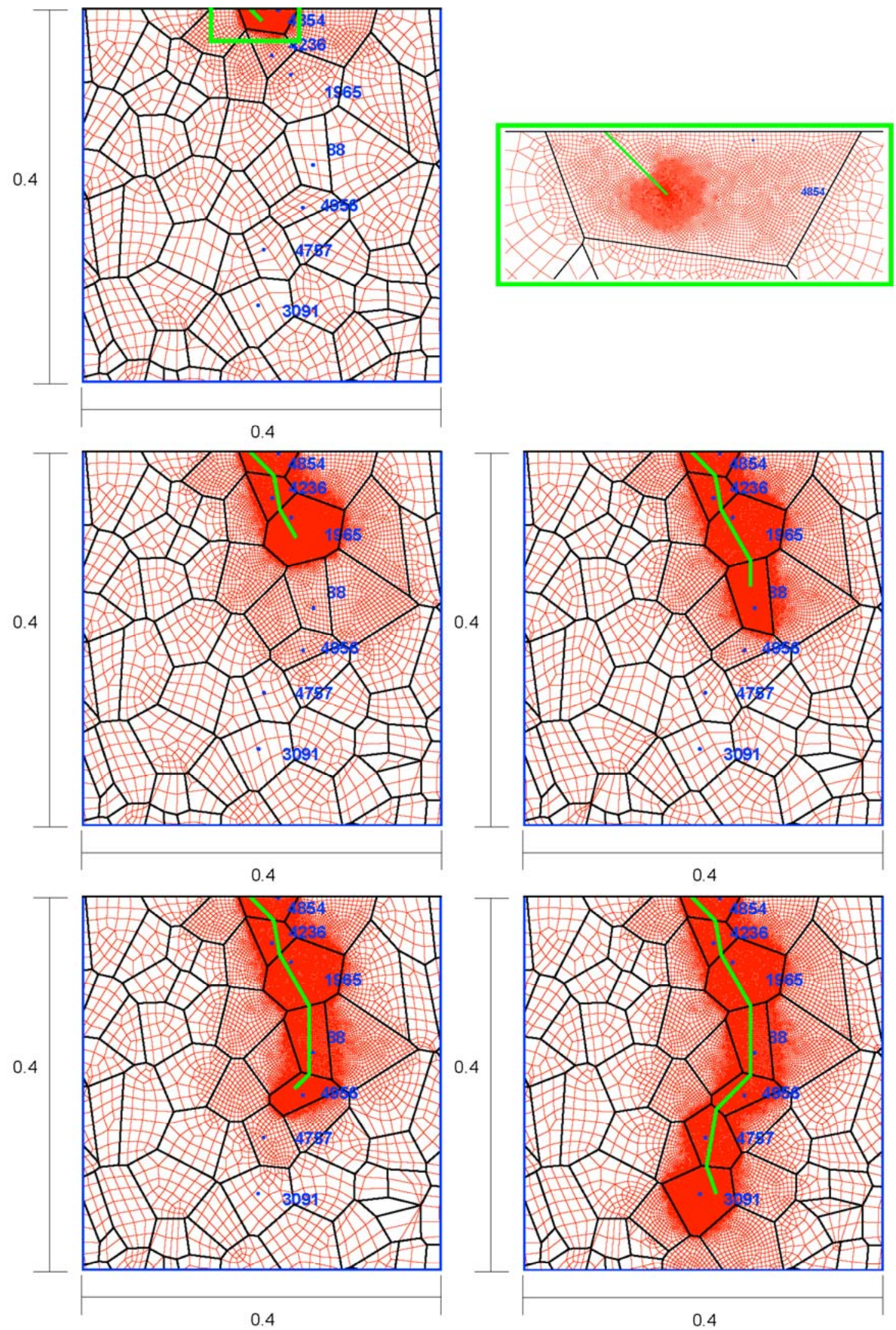

Figure 4 Details of the crack tip meshes for different crack lengths. Numbers indicate the grains containing the crack. Please refer to Figure 1 for the global position of the crack in the model. 
Table 1 Crack and crystal orientations and effective crack lengths

\begin{tabular}{|r|r|r|r|r|r|c|}
\hline \multirow{2}{*}{$\begin{array}{r}\text { Grain } \\
\text { No. }\end{array}$} & $\begin{array}{c}\text { Crystal } \\
\text { orientation } \\
\boldsymbol{\alpha}\left[{ }^{\circ}\right]\end{array}$ & $\begin{array}{c}\text { Crack } \\
\text { direction } \\
\boldsymbol{\theta}\left[{ }^{\circ}\right]\end{array}$ & \multicolumn{2}{|c|}{ Crack length [mm] } & \multirow{2}{*}{$\begin{array}{c}\text { CTOD } \\
\text { calculated }\end{array}$} \\
\cline { 5 - 7 } & 9.375 & 315 & 0.0187 & 0.0132 & 0.0093 & Yes \\
\hline 4236 & -25.264 & 280 & 0.0578 & 0.0465 & 0.0569 & No \\
\hline 1965 & -5.264 & 300 & 0.1114 & 0.0951 & 0.0929 & Yes \\
\hline $\mathbf{8 8}$ & $\mathbf{- 3 5 . 2 6 4}$ & $\mathbf{2 7 0}$ & $\mathbf{0 . 1 6 9 3}$ & $\mathbf{0 . 1 4 9 0}$ & $\mathbf{0 . 1 8 7 4}$ & Yes \\
\hline $\mathbf{4 9 5 6}$ & $\mathbf{- 8 0 . 2 6 4}$ & $\mathbf{2 2 5}$ & $\mathbf{0 . 2 3 9 8}$ & $\mathbf{0 . 2 1 2 7}$ & $\mathbf{0 . 1 4 9 5}$ & Yes \\
\hline 4757 & -45.264 & 260 & 0.2974 & 0.2609 & 0.3193 & No \\
\hline 3091 & -15.264 & 290 & 0.3671 & 0.3281 & 0.3694 & Yes \\
\hline
\end{tabular}

The shape of the crack assessed in this paper is rather complex. It is therefore useful to introduce a suitable effective crack shape with available linear elastic fracture mechanics (LEFM) solution. Two immediate purposes of the effective crack shape and length come in mind:

- Availability of reliable reference values of LEFM crack tip opening displacement (CTOD) and

- Proportionality of the CTOD to the crack length in LEFM.

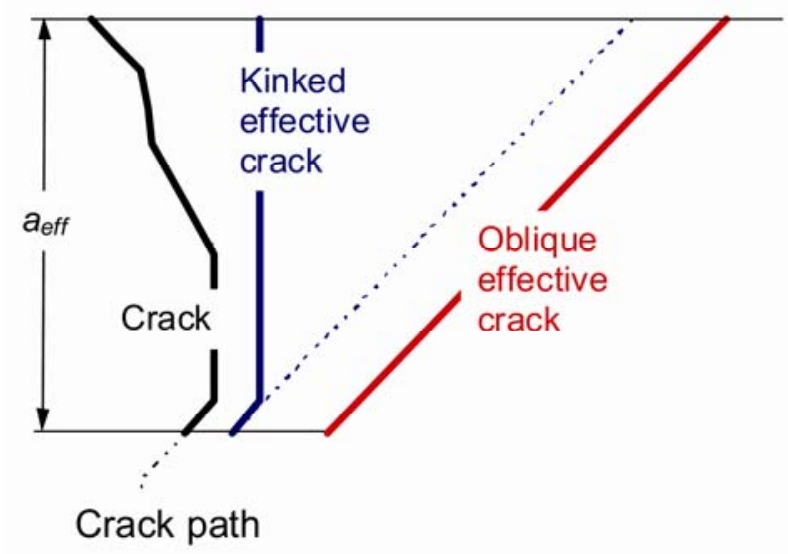

\section{Figure 5 Possible effective crack shapes and effective crack length}

Two crack shapes with available solutions [23] have been selected: kinked and oblique cracks emanating from a surface of a semi infinite plate, as depicted in Figure 5. It turns out that the difference in stress intensity factors between kinked and oblique cracks in similar configurations (see Figure 5) is of the order of $1 \%$ or less. Simpler oblique crack has been therefore selected as the representative effective crack. The appropriate lengths of effective 
cracks are listed in Table 1. It should be noted here that the physical crack length is not always a good measure of the CTOD: crack ending in grain No 88 is physically longer than crack ending in grain No 4956, but nevertheless results in much lower elastic CTOD.

\subsection{Crack tip opening displacement}

For each case the crack tip opening (CTOD) displacements are calculated at a distance of $2.5 \%$ of the average grain size behind the crack tip (i.e. $0.025 \cdot 52.9=1.3 \mu \mathrm{m}$ ) as depicted in Figure 6. Such definition of CTOD was taken from authors previous work where the effect of the grain boundary on the crack tip was evaluated [22]. Calculating CTOD at a larger distance behind the crack tip would diminish observable effect of the grain boundary and result in the linear increase of the CTOD with increased load, since the material far behind the crack tip is deformed elastically. The definition of the CTOD is also consistent with examples found in the literature[24],[25].

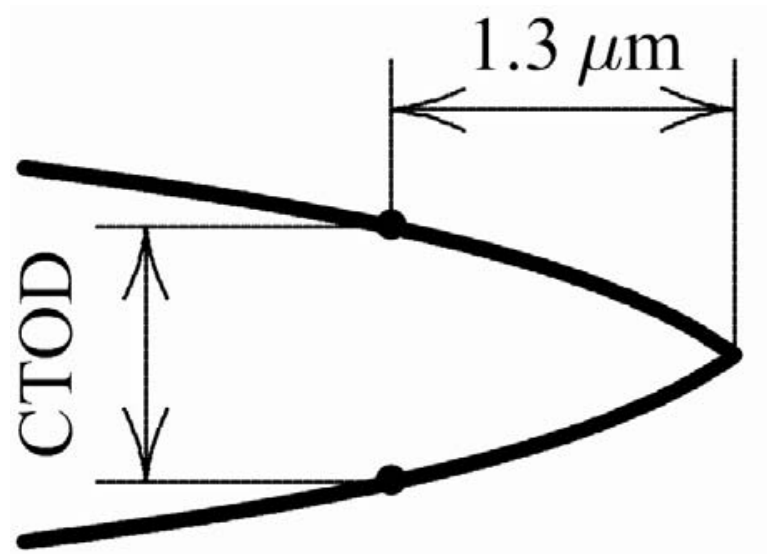

Figure 6 Definition of crack tip opening displacement CTOD 


\section{RESULTS}

Before embarking on the discussion of the results obtained with rather complex models containing randomly oriented crystal grains (monocrystals) it may be useful to give some insight in the constitutive response of anisotropic monocrystals. Changing the orientation of the lattice in the monocrystal with respect to the uniaxial load namely directly influences the stiffness of the monocrystal (individual grain) in the direction of the macroscopic loading (Figure 7, [13], $\sigma_{11}=240 \mathrm{MPa}$ ). The contribution from the anisotropic elasticity (dashed line in Figure 7) is notable, but moderate. The largest contribution comes from the crystal plasticity: firstly, the stiffness in crystal plasticity is defined by the Schmid factors (eq. 4) and secondly by the power law dependence of the shear rate, eq. (5). Due to the rather high exponent in eq. (5), directions with slightly smaller Schmid factors will exhibit significantly smaller shear rate. The combination of the two factors results in very distinct directions with stiff and soft response of the monocrystal.

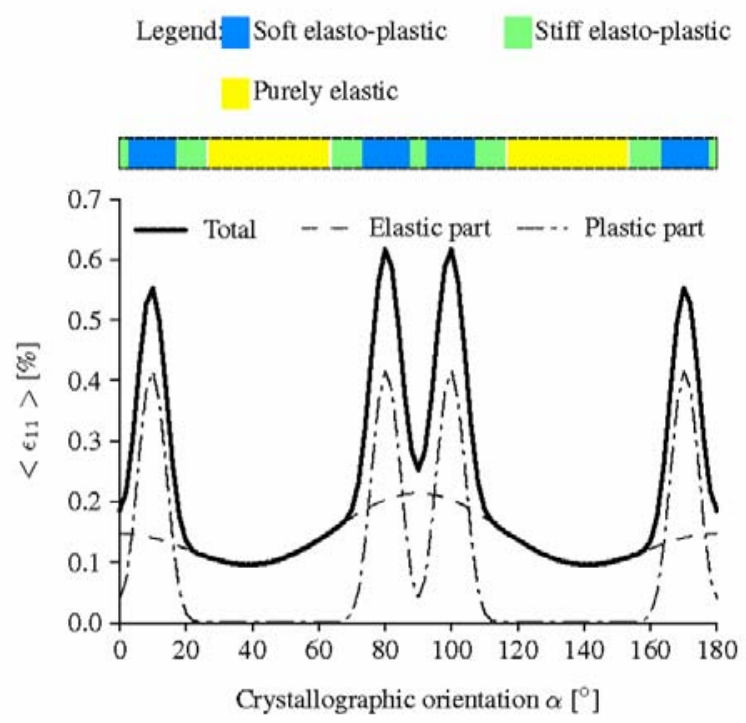

Figure 7 Strain $\varepsilon_{11}$ in a single crystal as a function of crystallographic orientation.

Three somewhat arbitrary levels of the stiffness in the directional response of the monocrystal are defined here for illustrative purposes: (1) purely elastic response, (2) stiff elastic-plastic with $0<=\varepsilon^{p l}{ }_{11}<0.1 \%$ and (3) soft elastic-plastic response with $0.1 \%<=\varepsilon^{p l}{ }_{11}$. Stiffness of the crack containing grains with respect to the uniaxial loading is depicted in Figure 8. It is noted here that the fourth and sixth grain on the crack path (Grains No. 88 and 4757) would, without a crack, remain elastic under the applied remote loading, and consequently represent notable resistance towards local deformation.

The role of grain orientations is basically twofold. Obviously, the "softness" of the grains in the vicinity of the crack has a major influence on the CTOD. Additionally, if clusters of 'soft' grains are present in the vicinity of the crack tip, the macroscopic bands of localized strain could coincide with the crack tip, resulting in large CTOD values. On the contrary, when clusters of 'soft' grains form far from the crack tip, the bands of localized strain could form elsewhere, which could significantly reduce the CTOD values [12]. Such effects could become even more pronounced in textured materials. 


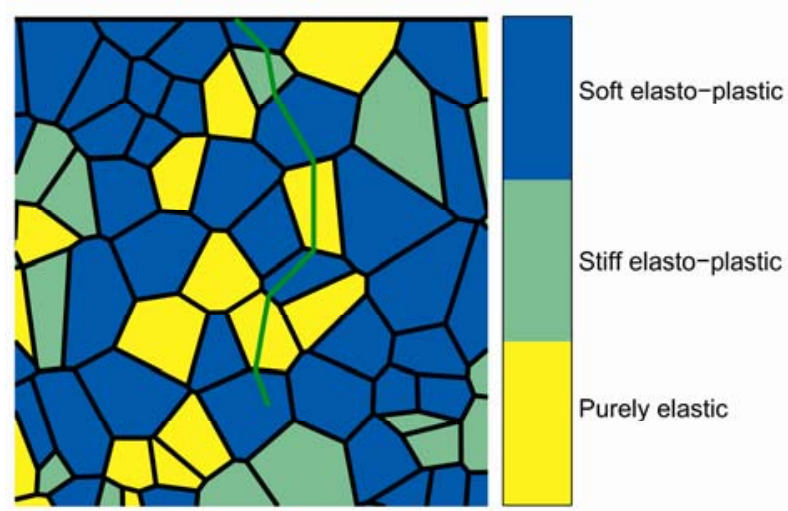

Figure 8 Grain stiffness for an arbitrarily selected set of crystallographic orientations. Orientations of the crack-containing grains were kept constant for all analyses reported in this paper.

A set of 30 models with different random grain orientations were generated for the five different crack lengths depicted in Figure 4 and detailed in Table 1. For each of the crack lengths, the average and standard deviation values of CTOD were then calculated. The 30 input values are sufficient to obtain statistically reasonably stable estimates of averages and standard deviations. It was expected that, with increasing crack length, the CTOD would depend less upon the surrounding microstructural features. Consequently, since the scatter of the CTOD is caused by the random orientations of the grains in the model, the value of the CTOD standard deviation is expected to decrease.

Figure 9 displays CTOD values as a function of effective crack length. The effective crack length is discussed in Section 2.3 Layout of cracks and has been introduced to minimize the impact of the crack shape on the discussion of the results. As expected, the CTOD values obtained using isotropic linear elastic material, are nearly proportional to the effective crack length. The small amount of non-proportionality is believed to be caused by rather complex crack shape, which could not be entirely captured by the effective crack approximation.

The mean values of the CTOD obtained using crystal plasticity and randomly oriented grains increase with the effective crack length. The exception is the stable CTOD value between the cracks with effective length of $0.1495 \mathrm{~mm}$ (crack tip in grain 4956) and $0.1874 \mathrm{~mm}$ (crack tip in grain 88). Please note here that the crack with effective crack length of $0.15 \mathrm{~mm}$ is physically longer than the crack with effective crack length of $0.19 \mathrm{~mm}$ (Table 1). In addition, the relative stiffness of the grain containing the tip of the crack with $a_{e f f}=0.19 \mathrm{~mm}$ is much higher than the relative stiffness of the grain containing the tip of the crack with $a_{\text {eff }}=0.15 \mathrm{~mm}$ (compare Figure 8).

The CTOD value obtained by the isotropic plasticity model is shown to represent an approximate lower limit. Both the isotropic elastic and isotropic plastic models are shown to significantly underestimate the CTOD values.

The scatter of the CTOD is indicated in Figure 9 with error bars denoting the range of \pm 3 standard deviations ( $\sim 99 \%$ of all random realizations) and increases with the effective crack length. 


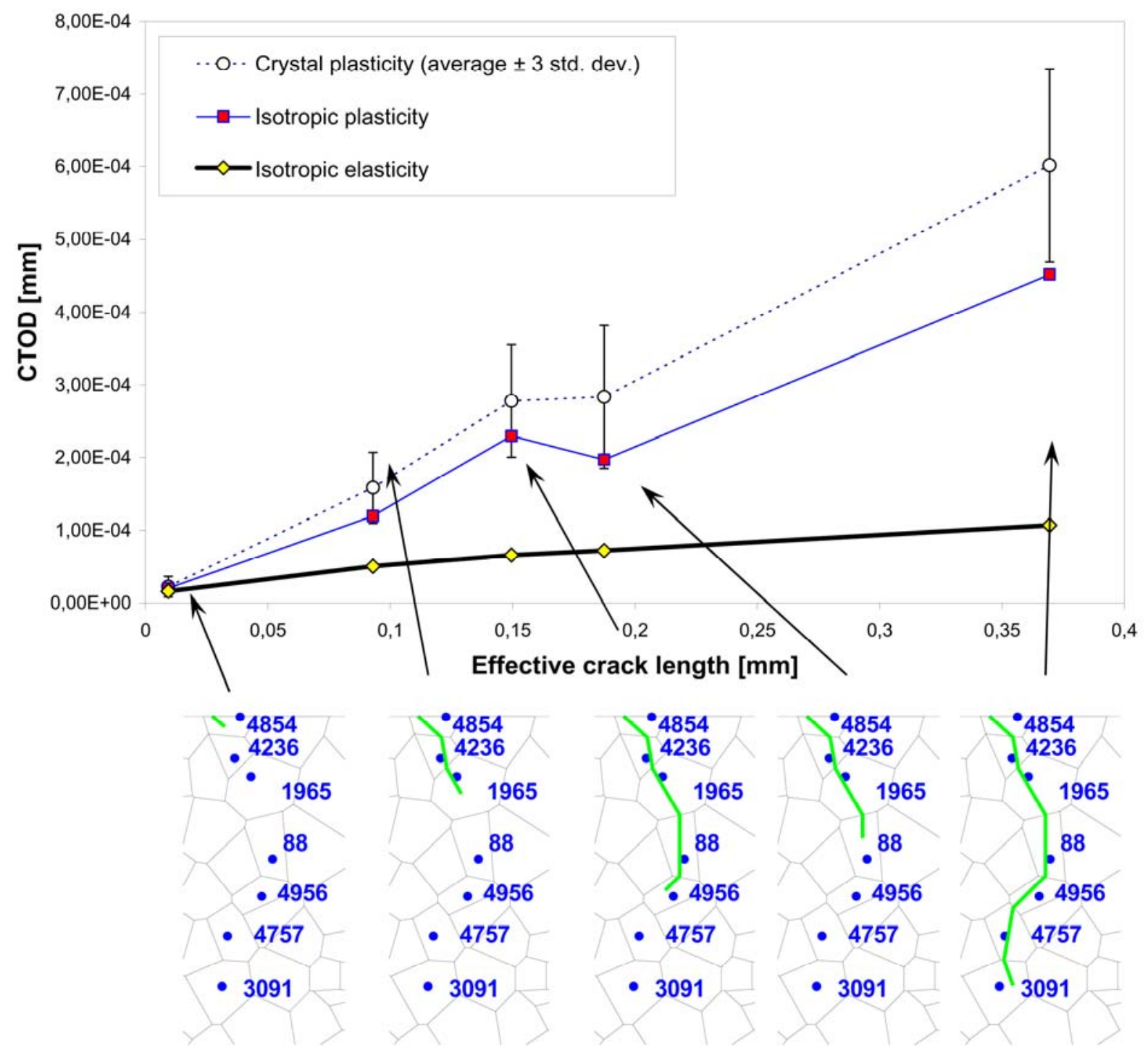

Figure 9 Scatter of the CTOD values due to the random nature of crystallographic orientations of the surrounding grains.

The normalized scatter of the CTOD is depicted in Figure 10 using the standard deviation as a measure of the scatter. Rather sharp decrease of scatter form above $20 \%$ to about $10 \%$ is noted for very short cracks. Further moderate decrease of scatter is seen with increasing the effective crack length. For the longest crack analyzed, which extends through seven grains, the standard deviation of the CTOD values is $7.3 \%$. 


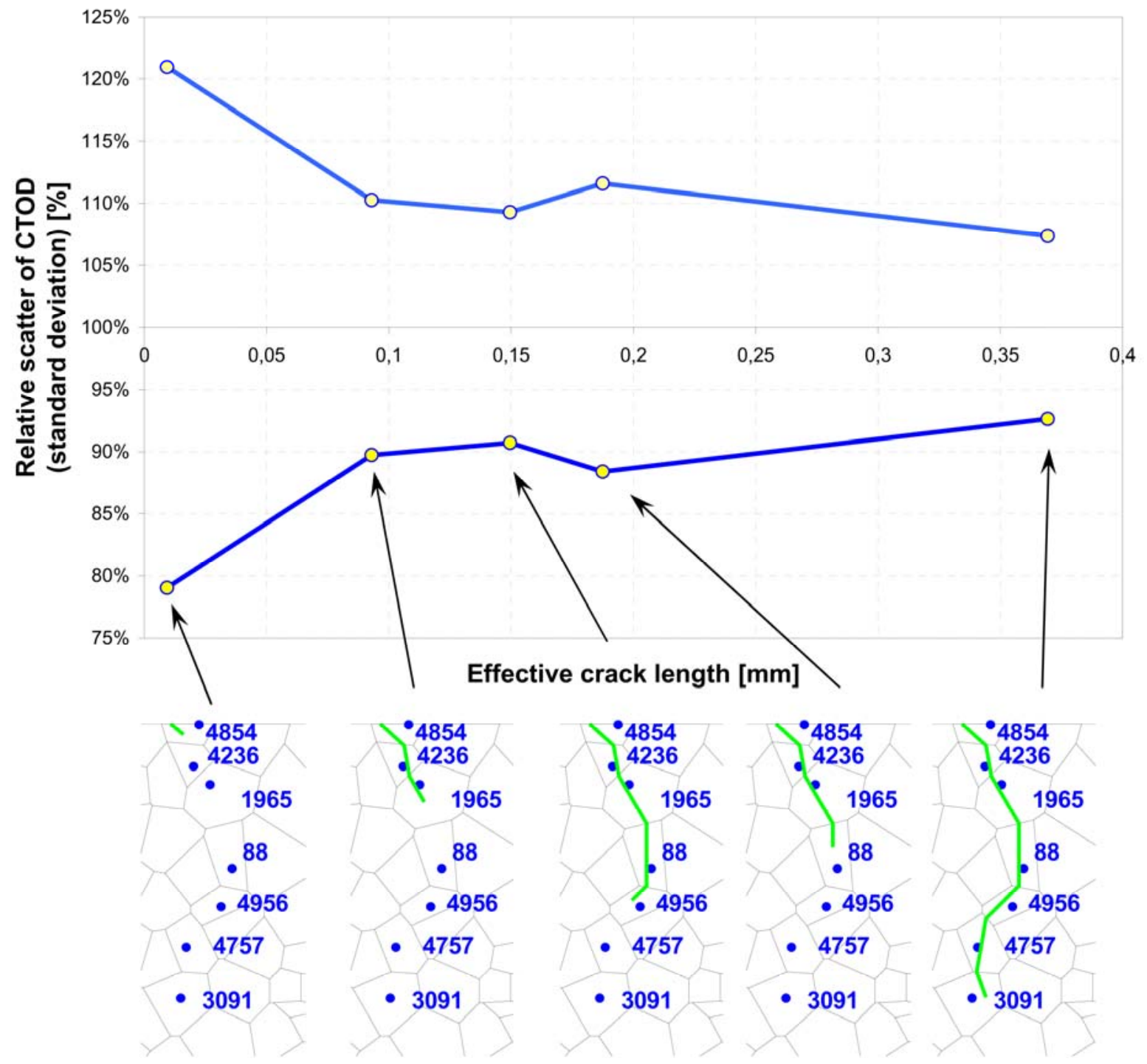

Figure 10 Normalized scatter of the CTOD values due to the random nature of crystallographic orientations of the surrounding grains.

The decrease of scatter was less pronounced than expected. Some possible reasons for this include:

- The crack was always placed in the P2 slip plane. In some cases, placing the crack in the $\mathrm{P} 4$ slip plane would result in the crack being more perpendicular to the external load, resulting in larger CTOD due to the remote load, which could mask some part of the scatter. This aspect was, however, not explored in this work. The emphasis was put on the evaluation of the scatter of the CTOD due to the crystallographic orientation.

- A micro-crack usually forms at surface extrusions/inclusions or along slip bands. The formation and position of these structures depends significantly on the crystallographic orientations of the grains. For different set of crystallographic orientations the position of these structures differs and the initial position of the crack should follow this. In our case the initial position of the crack is, however, fixed.

- The crack extending through 7 grains could be too short to become independent of the surrounding microstructural features. However, the longest crack in the model is longer than $10 \%$ of the model height. Longer cracks would require model with larger number of grains, which is currently beyond our computational capabilities. 
Based on the limited experience already gained with the 3D simulations [26], qualitatively similar results are expected for a 3D model, with possible quantitative change in the amount of scatter. Additional work is currently under way to verify this.

\section{CONCLUSIONS}

In this paper the approach to estimate the crack length with vanishing influence from the microstructural feature is proposed. This is achieved through a model containing a large number of randomly sized, shaped and oriented grains.

A series of transgranular cracks of lengths from 1 to 7 typical grain lengths are inserted into the model, extending from a grain at the surface and kinking across grain boundaries towards the interior of the model. Anisotropic elasticity and crystal plasticity constitutive models are employed at the grain size scale with grain boundaries modeled simply as a discontinuity in the lattice orientation between neighboring grains. Although the loading was monotonic, a number of key observations may be relevant for the fatigue case.

- The CTOD values obtained show rather high scatter. The isotropic plasticity model is shown to represent an approximate lower limit of those scattered values. Both the isotropic elastic and isotropic plastic models are shown to significantly underestimate the CTOD values.

- The scatter of the CTOD is measured using the standard deviation. It decreases rapidly from $20 \%$ for a crack embedded in a single grain to about $10 \%$ for cracks spanning 2 3 grains. Slower decrease to $7 \%$ for crack spaning seven grains is observed.

From the engineering point of view, cracks extending through more than about 10 grains may be analyzed with classical continuum mechanics approaches with reasonable accuracy. Shorter cracks may however require more sophisticated multiscale approaches.

The proposed approach is deemed useful for the understanding of the initiation and coalescence of the multiple stress corrosion cracks. A model of such cracks is currently under development with emphasis on the appropriate grain boundary properties, which have been disregarded in the presented work.

\section{ACKNOWLEDGEMENTS}

Financial support of the Slovene Research Agency (www.arrs.si) through grants P2-0026, J29168 and V2-0375 (co sponsored by the Slovene Nuclear Safety Administration www.ursjv.si) is gratefully acknowledged. 


\section{REFERENCES}

[1] Miller, K. J., 1987. "The behaviour of short fatigue cracks and their initiation. Part II-A general summary". Fatigue \& Fracture of Engineering Materials \& Structures, 10(2), pp. 93-113.

[2] Hussain, K., 1997. "Short fatigue crack behaviour and analytical models: a review". Engineering Fracture Mechanics,58(4), November, pp. 327-354.

[3] Hussain, K., de los Rios, E., and Navarro, A., 1993. "A two-stage micromechanics model for short fatigue cracks". Engineering Fracture Mechanics, 44(3), February, pp. 425-436.

[4] Morris, W. L., Buck, O., and Marcus, H. L., 1976. "Fatigue crack initiation and early propagation in A1 2219-T851". Metallurgical Transactions A, 7A, August, pp. 11611165.

[5] Zhang, Y.H., and Edwards, L., 1994. "On the blocking effect of grain boundaries on small crystallographic fatigue crack growth". Materials Science and Engineering A, 188(1-2), November, pp. 121-132.

[6] Düber, O., Künkler, B., Krupp, U., Christ, H.-J., and Fritzen, C.-P., 2006. "Experimental characterization and two-dimensional simulation of short-crack propagation in an austenitic-ferritic duplex steel”. International Journal of Fatigue, 28(9), September, pp. 983-992.

[7] Suresh, S., 1991. Fatigue of materials. Cambridge University Press.

[8] Tvergaard, V., Wei, Y., and Hutchinson, J. W., 2001. "Edge cracks in plastically deforming surface grains". European Journal of Mechanics-A/Solids, 20(5), SeptemberOctober, pp. 731-738.

[9] Zhai, T., Wilkinson, A. J., and Martin, J. W., 2000. "A crystallographic mechanism for fatigue crack propagation through grain boundaries". Acta Materialia, 48(20), December, pp. 4917-4927.

[10] Watanabe, T., 1984. "Approach to grain boundary design for strong and ductile polycrystals". ResMechanica :In-ternational Journal of Structural Mechanics and Materials Science, 11(1), pp. 47-84.

[11] Lehockey, E. M., Brennenstuhl, A. M., and Thompson, I., 2004. “On the relationship between grain boundary connectivity, coincident site lattice boundaries, and intergranular stress corrosion cracking”. Corrosion Science, 46(10), October, pp. 23832404.

[12] Simonovski, I., Nilsson, K., and Cizelj, L., 2007. "Crack tip displacements of microstructurally small cracks in 3161 steel and their dependence on crystallographic orientations of grains". Fatigue and Fracture of Engineering Materials and Structures (30), pp. 463-478.

[13] Simonovski, I., Nilsson, K.-F., and Cizelj, L., 2007. "The influence of crystallographic orientation on crack tip dis-placements of microstructurally small, kinked crack crossing the grain boundary". Computational Materials Science, 39(4), pp. 817-828.

[14] Kalinin, G., Barabash, V., Fabritsiev, S., Kawamura, H., Mazul, I., Ulrickson, M., Wu, C., and Zinkle, S., 2001. "ITER R\&D: Vacuum vessel and in-vessel components: Materials development and test". Fusion Engineering and Design, 55(2-3), July, pp. 231-246.

[15] Riesch-Oppermann, H., 1999.VorTess generation of 2- D random Poisson-Voronoi mosaics as framework for micromechanical modeling of polycrystalline materialsalgorithm and subroutines description. Tech. Rep. FZKA 6325, Forschungszentrum Karlsruhe. 
[16] Weyer, Stefan, Fröhlich, Andreas, Riesch-Oppermann, Heinz, Cizelj, Leon, Kovač, Marko, 2002. Automatic finite element meshing of planar Voronoi tessellations. Eng. fract. mech. (69) pp 945-958.

[17] Taylor, G. I. 1938. Plastic strain in metals. Journal of Institute of Metals, 62, 307-324.

[18] Hill, R., and Rice, J. R., 1972. "Constitutive analysis of elastic-plastic crystals at arbitrary strain". Journal of the Mechanics and Physics of Solids, 20(6), pp. 401-413.

[19] Rice, J. R., 1970. "On the structure of stress-strain relations of time-dependent plastic deformation in metals". Journal of Applied Mechanics,3 7, pp. 728-737.

[20] Huang,Y., 1991. A user-material subroutine incorporating single crystal plasticity in the ABAQUS finite element program. Tech. rep., Division of Applied Sciences, Harvard University (accessible through http://www.columbia.edu/1jk2079/fem/umat documentation.pdf).

[21] Peirce, D., Asaro, R. J., and Needleman, A., 1983. "Material rate dependence and localized deformation in crystalline solids". Acta Metallurgica, 31(12), December, pp. 1951-1976.

[22] Simonovski, I., Nilsson, K.-F., and Cizelj, L., 2005. "Material properties calibration for 316L steel using polycrystalline model". In 13th International Conference On Nuclear Engineering, May 16-20, 2005, Beijing, China.

[23] Murakami, Y., The Stress Intensity Factor Handbook, 1987, Pergamon Press.

[24] Potirniche, G. P., and Daniewicz, S. R., 2003. "Analysis of crack tip plasticity for microstructurally small cracks using crystal plasticity theory". Engineering Fracture Mechanics, 70(13), September, pp. 1623-1643.

[25] Bennett, V. P., and McDowell, D. L., 2003. "Crack tip dis-placements of microstructurally small surface cracks in single phase ductile polycrystals". Engineering Fracture Mechanics, 70(2), January, pp. 185-207.

[26] Cizelj, Leon, Simonovski, Igor. 2008. Simulated planar polycrystalls with planar and spatial random lattice orientatins. Proceedings of the 9th Biennal ASME Engineering Systems Design and Analysis Conference, July 7-9, 2008, Haifa, Israel. 


\section{LIST OF TABLES}

Table 1 Crack and crystal orientations and effective crack lengths

\section{LIST OF FIGURES}

Figure 1 The outline of the finite element model.

Figure 2 Relation between the slip systems of a face centered cubic crystal and the crack for crystallographic orientation $\alpha=0^{\circ}$ and crack direction $\theta=315^{\circ}$.

Figure 3 Angles between the slip planes and the crack plane for crystallographic orientation $\alpha=0^{\circ}$ and crack direction $\theta=315^{\circ}$.

Figure 4 Details of the crack tip meshes for different crack lengths. Numbers indicate the grains containing the crack. Please refer to Figure 1 for the global position of the crack in the model.

Figure 5 Possible effective crack shapes and effective crack length

Figure 6 Definition of crack tip opening displacement CTOD

Figure 7 Strain $\varepsilon_{11}$ in a single crystal as a function of crystallographic orientation.

Figure 8 Grain stiffness for an arbitrarily selected set of crystallographic orientations.

Orientations of the crack-containing grains were kept constant for all analyses reported in this paper.

Figure 9 Scatter of the CTOD values due to the random nature of crystallographic orientations of the surrounding grains.

Figure 10 Normalized scatter of the CTOD values due to the random nature of crystallographic orientations of the surrounding grains. 